\title{
Joint Replenishment and Delivery Problem with Resource Constraint for Deteriorating Item
}

\author{
Chengyan Li, Jun Gao and Chuang Wang \\ School of Computer Science and Technology \\ Harbin University of Science and Technology, Harbin 150080, China \\ chengyan@hrbust.edu.cn
}

\begin{abstract}
The joint replenishment and delivery model with deterministic resource restryetion for deteriorating item is developed. The model is formulated as cost minimization problem, including the ordering cost, the inventory holding cost, the transportation cost, the customer waiting cost and the deterioration cost. Differential evolution (DE) algorithm is proposed to solve the model. Numerical illustration of the model and algorithm are presented and the sensitivity analysis with respect to deteriorgtion rate of item is performed. The comparison between DE and genetic algorithn (GA) for solving the model are also made.
\end{abstract}

Keywords: Supply chain managencentyoint replenishment and delivery problem Resource constraint deteriorating item Differential velution

\section{Introduction}

For the past few decades, the supply chain management (SCM) has received much attention from the business community. Many companies have realized that significant cost saving can bo achieved by integrating inventory control and delivery policies throughout their supply chains, that is, the joint replenishment and delivery scheduling (JRD) polic. In KRD, cost can be saved when replenishment of several items are coordinated in a multi-iteh inventory system. Replenishment of a group of item takes place after a fixed interval of time, called the basic cycle time. Time between two consecutive orders of an item is assumed to be an integer multiple of the basic cycle time. The objective of the JRD is to minimize the total costs incurred per unit time.

Moon et al. [H] developed joint replenishment and consolidated freight delivery policies for a third party warehouse with deterministic demand rates in a supply chain. Cha et a D 2 dealt with the JRD model of the one-warehouse n-retailer system and suggested a flexible policy for a warehouse. Wang et al. [3] studied a joint replenishment and de ivery scheduling in which a central warehouse serves n-retailers in the presence of vague operational conditions such as ordering cost and inventory holding cost, and the membership function is approximated using piecewise linear functions based on alpha level sets.

The deteriorating items are subject to a continuous loss in their masses or utility throughout their lifetime due to decay, damage, spoilage, and penalty of other reasons, and most of the physical goods undergo decay or deterioration over time [4]. Considering to this fact, controlling and maintaining the inventory of deteriorating items becomes a challenging problem for decision makers. Chaman developed two-warehouse supply chain model with power form stock-dependent demand under the assumption that the deterioration rate per unit items are different due to different preservation environments [5]. XU et al. considered production-inventory models for a deteriorating item in a single vendor-buyer system with constant production and demand rate, and developed Ant Colony algorithm to solve the problem [6]. Debasis et al. considered two-warehouse 
inventory model for a deteriorating item with time-varying demand and fully backlogged shortages, and using GA with vary population size to solve the model [7].

The differential evolution (DE) algorithm is one of the latest evolutionary optimization methods proposed by Storn and Price [8] for complex continuous non-linear functions. $\mathrm{DE}$ is a stochastic population-based optimization method which uses mutation, crossover, and selection operators at each generation to move its population toward the global optimum. Over the past ten years, many research indicated that the DE algorithm can solve the problem more effectively. Kazemipoor et al. [9] considered the multiskilled project portfolio scheduling problem and presented an efficient metaheuristic algorithm based on DE, the comparison between the results of DE and Tabu search confirms the effectiveness of the DE algorithm. Wang et al. developed an approach based on DE to find a close to optimum for the basic JRP [10]. Das and Suganthan [11] surveyed the state-of-the-art of the differential evolution and its application.

The aim of this paper is to develop a practical constraint JRD model with deteriorating item based on the early work. Secondly, for solving the problem, a DE algorithm is applied. Thirdly, to illustrate the effectiveness and efficiency of the algorithms, extensive computational experiments are performed.

The rest of this paper is organized as follows Section 2 introduces the mathematical model of constraint JRD with deteriorating item. Section 3 develops the DE algorithm and presents the procedure to solve the problem Section 4 illustrates the procedure of proposed algorithms with a numerical example. Section ssummarize the conclusions of the present work and provide directions for future research.

\section{Mathematical Model of Joint Replenishment and Delivery Problem with Deteriorating Item}

The constraint joint replenishment and deliwery problem with deteriorating item (which is abbreviated as Deter-C RD for the rest of the paper) is the multi-item inventory problem of coordinating the replenishment and delivery of a group of deteriorating items that may be jointly ordered from a single supplier under resource restrictions.

Some common assumptions usually made for the constrained joint replenishment problem:

- The demand rate of each item is deterministic and constant.

- The unit holding cost of each item is known and constant.

- The major ordering cost incurred for an order is known and constant.

- The minor ordering cost incurred for a specific ordered item is known and constant.'

- The outbound transportations cost incurred for a specific delivered item is known and constant.

The customer waiting cost is known and constant.

No quantity discount.

- Stock replenishment is complete when it occurs.

- The budget constraint on the amount of an order is known and constant.

- The deterioration rate of item follows exponential distribution.

The following notation is defined:

$i \quad$ index of item, $i=1,2, \ldots, n$

$D_{i} \quad$ demand rate of item $i$

$S^{W}$ major ordering cost

$s_{i}{ }^{W}$ minor ordering cost when item $i$ is included in a group replenishment

$h_{i}{ }^{W}$ inventory holding cost of item $i$, per unit per unit time

$s_{i}^{C}$ outbound transportation cost for item $i$

$w_{i}{ }^{C}$ customer waiting cost for item $i$ per unit, per unit time

$T$ basic cycle time (decision variable) 
$k_{i} \quad$ integer number that decides the replenishment schedule of item $i$ (decision variable)

$K \quad n \times 1$ vector that consists of $k_{i}, i=1,2, \ldots, n$

$f_{i}$ integer number that determines the outbound delivery schedule of item $i$ (decision variable)

$F \quad n \times 1$ vector that consists of $f_{i}, i=1,2, \ldots, n$

$T C$ total annual cost for all items, objective function that is to be minimized

$b_{i} \quad$ unit cost of item $i$

$B \quad$ limit on capital that can be invested

$r_{i} \quad$ unit deterioration cost of item $i$

$\theta_{i} \quad$ deterioration rate of item $i$.

The initial inventory level is $I_{0}$ at time 0 . From $t=0$ to $T$, the inventory level Teduces, owing to both demand and deterioration, until it reaches zero level. At this time, shortage is accumulated and backlogged. At the end of the cycle, the inventory yeplerishes and again raises the level to $I_{0}$, as shown in Figure 1 .

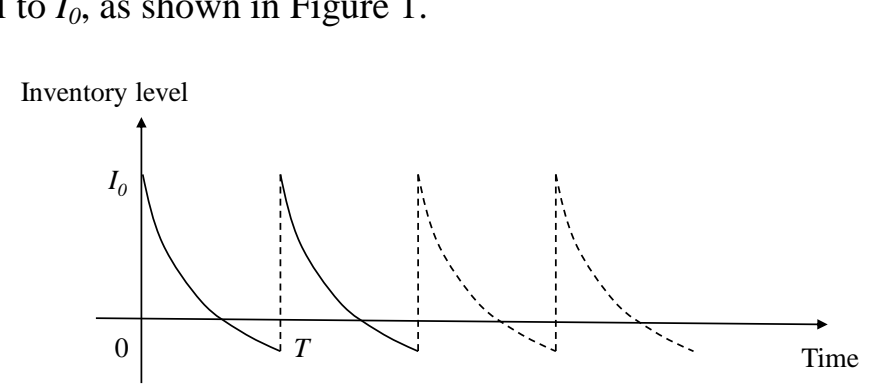

\section{Figure 1. Graphical Representation of Inventory Level}

The inventory level attime $t$ is governed by the Eq. (1).

Then

$$
\frac{d I_{i}(t)}{d t}+\theta_{i} I_{i}(t)=-D_{i}, I_{i}(T)=0
$$

$$
I_{i}(t)=\frac{B}{\theta_{i}}\left(e^{\theta_{i}(T-t)}-1\right)
$$

The inventory høing cost and deterioration cost of item $\mathrm{i}$ in basic replenishment cycle is

$$
h_{i}^{W} \int_{0}^{T} I_{i}(t) d t=h_{i}^{W} \int_{0}^{T} \frac{D_{i}}{\theta_{i}}\left(e^{\theta_{i}(T-t)}-1\right) d t=\frac{1}{2}\left(\frac{D_{i} h_{i}^{W} k_{i}\left(f_{i}-1\right) T}{f_{i}}+D_{i} \theta_{i} r_{i} k_{i} T\right)
$$

The model for constraint joint replenishment and delivery problem with deteriorating item is given by

(Deter-CJRD)

$$
T C(T, K, F)=\frac{S^{W}+\sum_{i=1}^{n} \frac{s_{i}^{W}}{k_{i}}}{T}+\sum_{i=1}^{n} \frac{D_{i} h_{i}^{W} k_{i}\left(f_{i}-1\right) T}{2 f_{i}}+\sum_{i=1}^{n} \frac{f_{i} s_{i}^{c}}{k_{i} T}+\sum_{i=1}^{n} \frac{k_{i} T D_{i} w_{i}^{c}}{2 f_{i}}+\sum_{i=1}^{n} D_{i} \theta_{i} r_{i} k_{i} T
$$

Subject to

$$
\begin{gathered}
\sum_{i=1}^{n} D_{i} k_{i} T b_{i} \leq B \\
k_{i} \in Z^{+}, f_{i} \in Z^{+}, T \in R^{+}, i=1,2, \ldots n
\end{gathered}
$$

Eq. (4) is the total cost to be minimized, that is the sum of the ordering cost (major and minor), the inventory holding cost, the outbound transportation cost, the customer waiting cost and the deterioration cost. The resource constraint is the limit on capital that can be 
invested, as given by Eq. (5). Eq. (6) indicates that $k_{i}^{\prime}$ 's and $f_{i}^{\prime}$ s are positive integer number and $T$ is positive real number.

To solve the unconstrained JRD, Moon et al. used Eq. (7) as the optimal $T$ in their algorithm.

$$
T^{*}=\left\lfloor 2\left(S^{W}+\sum_{i=1}^{n} \frac{s_{i}^{W}+f_{i} s_{i}^{C}}{k_{i}}\right) / \sum_{i=1}^{n} k_{i} D_{i}\left(h_{i}^{W}+\frac{w_{i}^{C}-h_{i}^{W}}{f_{i}}\right)\right]^{1 / 2}
$$

The optimality condition of $k_{i}$ is:

$$
k_{i}\left(k_{i}-1\right) \leq \frac{2\left(s_{i}^{W}+f_{i} s_{i}^{c}\right)}{T^{2} D_{i}\left(h_{i}^{W}+\frac{w_{i}^{C}-h_{i}^{W}}{f_{i}}\right)} \leq k_{i}\left(k_{i}+1\right)
$$

Similarly, the optimality condition of $f_{i}$ is :

$$
f_{i}\left(f_{i}-1\right) \leq \frac{k_{i}^{2} T^{2} D_{i}\left(w_{i}^{c}-h_{i}^{W}\right)}{2 s_{i}^{c}} \leq
$$

\section{Differential Evolution Algorithm}

In order to solve the constraint joint replenishment and delivery problem with deteriorating item, in this section, we present a differeptial evolution (DE) algorithm approach for the Deter-CJRD model. DE algorithm differing from conventional evolutionary optimization methods, stuch as genetic algorithm (GA), relies on the mutation operations as the main operator. The DE algorithm introduces a novel mutation operation which is simple and effective. The mutation operation is based on the differences of randomly sampled pairs of solution in the population. Furthermore, the fitness of an offspring is one-to-one competed with that of the corresponding parent in DE algorithm.

\subsection{Representation and Initialization}

The DE a goritm for the Deter-CJRD is to find an optimal schedule which can get a close to minimum total cost. The appropriate representation of a solution plays an important role in the development of DE algorithm. In the Deter-CJRD model, the basic cycle $T$ ang $n$ integer $k_{i}$ 's and $f_{i}$ 's have to be decided for solving the problem. In the $\mathrm{DE}, k_{i}$ 's and $f_{i}$ 's are searched through the operations of $\mathrm{DE}$ and the basic cycle $T$ is determined through the optimality condition of $T$. For the given $k_{i}$ 's and $f_{i}$ 's, the optimal $T^{*}$ can be easily obtained by the Eq. (7). So, in our study, we use $2 n$ random number representation for $n k_{i}$ 's and $n f_{i}^{\prime}$, s, because it is very easy to decode our chromosome to a feasible solution. As shown in Figure 2, the decoding indiridual contains two parts. One is for the replenishment schedule and the other is for the outbound schedule of each item.

\begin{tabular}{|c|c|c|c|c|c|c|c|c|c|c|c|}
\hline 1 & 1 & 1 & 2 & 2 & 4 & 4 & 3 & 2 & 3 & 2 & 2 \\
\hline \multicolumn{3}{|c|}{$\begin{array}{c}\text { Replenishment } \\
\text { schedule } k_{i}^{\prime} \text { 's }\end{array}$} & \multicolumn{6}{|c|}{$\begin{array}{c}\text { Outbound } \\
\text { schedule } f_{i} \text { 's }\end{array}$} \\
\hline
\end{tabular}

\section{Figure 2. Decoding Individual}

The initial population is created by assigning random integer values of the decision variable. Each individual is generated by Eq. (10).

$$
x_{i j}=\operatorname{rand}(), i=1,2, \ldots, P O P S I Z E, j=1,2, \ldots, D
$$

Where POPSIZE is the number of individuals; $D$ is the dimension of each individuals; $\operatorname{rand}()$ is a function which generates a uniform distribution rand number 
in range $\left[k_{i}{ }^{L B}, k_{i}{ }^{U B}\right]$ or $\left[f_{i}^{L B}, f_{i}^{U B}\right] . \quad k_{i}^{L B}$ and $k_{i}^{U B}$ are the lower and upper bound of $k_{i}$, respectively, and can be defined from the following equations.

$$
k_{i}^{L B}=1, k_{i}^{U B}=\left\lceil\frac{\sqrt{2\left(S^{W}+s_{i}^{W}\right) / D_{i} h_{i}^{W}}}{T_{\min }}\right\rceil
$$

$f_{i}^{L B}$ and $f_{i}^{U B}$ is the lower and upper bound of $f_{i}$, respectively, and can be defined from the following equations.

$$
f_{i}^{L B}=1, f_{i}^{U B}\left(f_{i}^{U B}-1\right) \leq \frac{\left(k_{i}^{U B}\right)^{2} T_{\max }^{2} D_{i}\left(w_{i}^{C}-h_{i}^{W}\right)}{2 s_{i}^{C}} \leq f_{i}^{U B}\left(f_{i}^{U B}+1\right)
$$

$T_{\max }$ and $T_{\min }$ are defined as follows

$$
\begin{aligned}
T_{\max } & =\left\lfloor 2\left(S^{W}+\sum_{i=1}^{n} s_{i}^{W}\right) / \sum_{i=1}^{n} D_{i} h_{i}^{W}\right]^{1 / 2} \\
T_{\min } & =\min \left(2 s_{i} / D_{i} h_{i}^{W}\right)
\end{aligned}
$$

For a given particular set of $k_{i}$ 's and $f_{i}$ 's, eqs. (4) and (5) can be written as

$$
T C(T)=\frac{C_{1}}{T}+C_{2} T,
$$

Subject to

Where

$$
C_{3} T \leq B
$$

$$
\begin{aligned}
& C_{1}=S^{W}+\sum_{i=1}^{n} \frac{s_{i}^{W}}{k_{i}}+\sum_{i=1}^{n} \frac{f_{i} s_{i}^{c}}{k_{i}}, C_{2} \sum_{i=0}^{n} \frac{\left(f_{i}-1\right){ }^{2} f_{i} h_{i}^{W}}{f_{i}}+\sum_{i=1}^{n} \frac{k_{i} D_{i} w_{i}^{c}}{2 f_{i}}+\sum_{i=1}^{n} D_{i} \theta_{i} r_{i} k_{i} \text { and } \\
& C_{3}=\sum_{i=1}^{n} D_{i} k_{i} b_{i} \text { are constants. }
\end{aligned}
$$

We take the first order derivative with respect to $T$, and set it to zero. Then we obtain $T^{0}=\sqrt{C / C_{2}} \wedge$ Ad for a constraint, we obtain $T_{1}=B / C_{3}$. According to Moon et al. [1], the optimal basic cycle time $T$ follows $T^{*}=\min \left(T^{0}, T^{1}\right)$.

\subsection{Mutation}

For each target individual $X_{i}=\left\{x_{i 1}, x_{i 2}, \ldots, x_{i D}\right\}, i=1,2, \ldots, P O P S I Z E$, a mutant new individual $b_{i}$ is generated according to

$$
V_{i}=X_{r 1}+F\left(X_{r 2}-X_{r 3}\right), r_{1} \neq r_{2} \neq r_{3} \neq i
$$

With randomly chosen integer indexes; $F \in[0,2]$ is a real number called mutation factor used to control the amplification of the differential variation.

\subsection{Crossover}

To complement the differential mutation search strategy, DE employs uniform crossover, which also known as binomial method, to enhance the potential diversity of the population. The crossover operator implements a discrete recombination of the trial individual $V_{i}$ and the parent individual $X_{i}$ to produce the offspring $X_{i}^{\text {new }}$.

The trial vector will be found using the following rules:

$$
x_{i j}^{n e w}=\left\{\begin{array}{l}
v_{i j} \text { if } \text { rand }_{j}(0,1) \leq C R \text { or } j=\operatorname{rnb}(i) \\
x_{i j} \text { otherwise } j=1,2, \ldots, D
\end{array}\right.
$$


Where $D$ is the dimension of $X_{i} ; x_{i j}$ refers to the $j$ th element of the individual; $v_{i j}$ is similarly defined; $\operatorname{rand}_{j}(0,1)$ is the $j$ th evaluation of a uniform random number generator between $[0,1] ; \operatorname{rnb}(i)$ is a randomly chosen integer in the set $\{1,2, \ldots, D\}$ which ensures the trail vector gets at least one parameter from the mutated vector; $C R \in[0,1]$ is a crossover constant.

\subsection{Selection}

The selection operator is to determine whether the target (parent) or the new vector (offspring) survives to the next generation. If a new vector, $X_{i}^{\text {new }}, i=1,2, \ldots, P O P S I Z E$, has a smaller evaluation function value (total cost) than its garget vector, $X_{i}$, it is copied to the next generation; otherwise, it is the target vector that passes to the next generation. The selection process can be expressed as:

$$
X_{i}= \begin{cases}X_{i}^{n e w} & \text { if } \operatorname{fitness}\left(X_{i}^{\text {new }}\right)<\operatorname{fitness}\left(X_{i}\right) \\ X_{i} & \text { otherwise }\end{cases}
$$

Thus the next generation of population either gets better in terns of the fitness function or remains constants.

\subsection{Stop Criterion}

The termination condition is to stop if no improverment of fitness function is made in 50 generations.

\section{Numerical Example}

A numerical example will be employed to illustrate the proposed DE algorithm, we use the numerical example of Moon et. al., [1] and Wang et. al., [3]. The data for this example are given in Table $1 \times$ We also assume $S^{W}=200$ and $B=25000$. Notice that, there are resource restriction, $b_{i}$ dnd $B$, in our example.

\section{Table 1. Parameter Values for the Example}

\begin{tabular}{lllllll}
\hline Item $i$ & & 3 & 3 & 4 & 5 & 6 \\
\hline$D_{i}$ & 10000 & 5000 & 3000 & 1000 & 600 & 200 \\
$s_{i}{ }^{W}$ & 45 & 46 & 47 & 44 & 45 & 47 \\
$h_{i}{ }^{W}$ & 1 & 1 & 1 & 1 & 1 & 1 \\
$s_{i}{ }^{C}$ & 5 & 5 & 5 & 5 & 5 & 5 \\
$w_{i}{ }^{C}$ & 1.5 & 1.5 & 1.5 & 1.5 & 1.5 & 1.5 \\
$b_{i}$ & 6.25 & 6.25 & 6.25 & 6.25 & 6.25 & 6.25 \\
$r_{i}$ & 1 & 1 & 1 & 1 & 1 & 1 \\
\hline
\end{tabular}

The DE algorithm is compared to the GA algorithm for the same numerical example. The results are reported in Table 2. It shows in Table 2 that DE obtains better solution than GA does. 
Table 2. Computational Results under Different $\Theta$

\begin{tabular}{|c|c|c|c|c|c|c|c|c|}
\hline \multirow{2}{*}{$\theta$} & \multicolumn{4}{|c|}{$\overline{D E}$} & \multicolumn{4}{|c|}{$\overline{\mathrm{GA}}$} \\
\hline & $k_{i}$ & $\overline{f_{i}}$ & $T$ & $T C$ & $k_{i}$ & $f_{i}$ & $T$ & $T C$ \\
\hline 0.00 & $1,1,1,2,2,4$ & $4,3,2,3,2,2$ & 0.1881 & 4831.71 & $1,1,1,2,2,4$ & $4,3,2,3,2,4$ & 0.1818 & 4836.37 \\
\hline 0.02 & $1,1,1,2,2,4$ & $4,3,2,3,2,2$ & 0.1881 & 4911.71 & $1,1,1,2,2,5$ & $4,3,2,3,2,8$ & 0.1802 & 4936.51 \\
\hline 0.04 & $1,1,1,2,2,4$ & $4,3,2,3,2,2$ & 0.1881 & 4991.71 & $1,1,1,2,2,3$ & $4,3,2,3,2,1$ & 0.1830 & 4998.02 \\
\hline 0.06 & $1,1,1,2,2,4$ & $4,3,2,3,2,2$ & 0.1881 & 5071.15 & $1,1,1,2,2,3$ & $4,3,2,3,2,4$ & 0.1800 & 5076.10 \\
\hline 0.08 & $1,1,1,2,2,4$ & $4,3,2,3,2,2$ & 0.1881 & 5149.38 & $1,1,1,2,2,4$ & $4,3,2,3,2,5$ & 0.1775 & 5159.95 \\
\hline 0.10 & $1,1,1,2,2,4$ & $4,3,2,3,2,2$ & 0.1881 & 5226.43 & $1,1,1,2,2,5$ & $4,3,2,3,2,9$ & 0.1743 & 5258.59 \\
\hline
\end{tabular}

We compare the performance of two algorithms, DE and GA, for 1600 randomby generated Deter-CJRDs. The parameter values are all generated from a uniform distribution, $D_{i} \sim \mathrm{U}[500,5000], s_{i}{ }^{W} \sim \mathrm{U}[30,50], s_{i}{ }^{C} \sim \mathrm{U}\left[0.1 s_{i}{ }^{W}, 0.3 s_{i}{ }^{W}\right], h_{i}{ }^{W} \sim \mathrm{U}[0.2,3.0]$ and $w_{i}{ }^{C} \sim \mathrm{U}\left[1.2 h_{i}{ }^{W}, 2.0 h_{i}{ }^{W}\right]$ respectively. Every $b_{i}$ is considered as $6.25, \quad i=1,2, \ldots, n$. Moreover, $\theta=0.02$ is constant. Four different values of the number of items, $n=10$, 20, 30 and 50, and four different values of the major ordering cost $S^{W}=100,200$, 300 and 400 are considered. This results in 16 combinations of th and $S^{W}$, and for each combination, 100 problems with random parameter values are generated and solved. The termination condition is to stop if no improvement is made in 50 generations.

A summary of computational results is shoun in Table 3.

Table 3. Comparison ofDE and GA

\begin{tabular}{|c|c|c|c|c|c|c|}
\hline \multirow[t]{2}{*}{$n$} & \multirow[t]{2}{*}{$S^{W}$} & \multicolumn{2}{|c|}{ Best solution problems } & \multicolumn{3}{|c|}{ DE better than GA (\%) } \\
\hline & & $\mathrm{DE}$ & & Num & Max & Avg. \\
\hline 10 & 100 & 100 & 100 & 80 & 5.2648 & 0.7359 \\
\hline & & & 100 & 83 & 1.9952 & 0.5502 \\
\hline & & 400 & 100 & 86 & 3.3217 & 0.7789 \\
\hline & & & 100 & 90 & 4.4830 & 0.7540 \\
\hline & & 100 & 100 & 86 & 4.4388 & 1.3800 \\
\hline & & 100 & 100 & 80 & 4.6668 & 0.6221 \\
\hline & 30 & 100 & 100 & 76 & 3.0064 & 0.6071 \\
\hline & 400 & 100 & 100 & 71 & 2.1448 & 0.6581 \\
\hline 30 & 100 & 100 & 100 & 82 & 3.2284 & 1.5014 \\
\hline & 200 & 100 & 100 & 83 & 4.6256 & 1.2560 \\
\hline & 300 & 100 & 100 & 81 & 4.4656 & 0.7110 \\
\hline & 400 & 100 & 100 & 76 & 2.0580 & 0.6614 \\
\hline & 100 & 100 & 100 & 93 & 1.8431 & 0.9628 \\
\hline & 200 & 100 & 100 & 98 & 3.9306 & 2.5099 \\
\hline & 300 & 100 & 100 & 100 & 5.4950 & 3.3884 \\
\hline & 400 & 100 & 100 & 99 & 6.9276 & 3.9919 \\
\hline
\end{tabular}

As show in Table 3, both algorithms can solve the 1600 random generated problems. The DE is superior to GA algorithm, especially for $n=50$.

\section{Conclusion}

This paper focuses on the constrained joint replenishment and delivery problem with deteriorating item, and developing efficient algorithms to solving it. We introduced the $\mathrm{DE}$ algorithm to handle the problem. Using comprehensive computational experiments, we have shown the performance of the DE. Furthermore, DE is more suitable for solving 
Deter-CJRD than the GA algorithm. Future research could be the incorporation of uncertainty issue in constrained JRD, for example demand and budget.

\section{Acknowledgments}

This work was supported by grant No.12541142 from the Research Program of the Education Department of Heilongjiang Province, China.

\section{References}

[1] K. Moon, B. C. Cha and C. U. Lee, "The joint replenishment and freight consolidation of a warehouse in a supply chain”, Int. J. Prod Econ. vol. 1, no. 133, (2011).

[2] B. C. Cha, I. K. Moon and J. H. Park, "The joint replenishment and delivery scheduling of the onewarehouse, n-retailer system", Transport Res E-Log., vol. 5, no. 44, (2008).

[3] L. Wang, D. CX, C. G. Lee, Q. L. F and Y. R. Z, Model and algorithm for fuzzy joint replepishment and delivery scheduling with explicit membership function. Int. Journal of Adv Manuf. rol. 66, (2013), pp. 9-12.

[4] V. K. Mishra, L. S. Singh and R. Kumar, "An inventory model for deteriorating items with timedependent demand and time-varying holding cost under partial backlogging", Journal of Industrial Engineering Int., vol. 4, no. 9, (2013).

[5] C. Singh and S. R. Singh, "Optimal ordering policy for deteriorating items with power-form stock dependent demand under two-warehouse storage facility", OP search, vol. 2, no. 50, (2013).

[6] H. Xu, C. Y. Li, and L. B. Zhou, "Research on order problem for perishable products based on ant colony algorithms", J. Harbin University Of Science and Technoløgy, vol. 3, no. 15 (2010).

[7] D. Debasis, B. K. Mohuby, R Arindam and K Samarjit, "Two-warehouse production inventory model for a deteriorating item with time-varying demand and shortages: a genetic algorithm with varying population size approach", Optimal Engineering, vol.,15, (2014).

[8] R. Storn and K. K. Price, "Differential evolution- a sinple and efficient heuristic for global optimization over continuous spaces", J. Glob Optim, vol. 4, no. 11 (1997).

[9] H. Kazemipoor, R. T. Moghaddan. P. S. Shahrezaei and A. Azaron, "A differential evolution algorithm to solve multi-skilled project portfolio scheduling problems", Int. Journal of Advance Manufacture Technology, vol. 5-8, no. 64, (2013).

[10] L. Wang, J. He and Y R. Zeng, "A differential evolution algorithm for joint replenishment problem using direct grouping and its application", Expert Syst., vol. 5, no. 29, (2012).

[11] S. Das and P. N. Suganthan, "Differential evolution: A survey of the state-of-the-art", IEEE T Evolution Computer, vol. 15.(2011). 